\title{
A RANDOM FIXED POINT THEOREM FOR MULTIVALUED NONEXPANSIVE OPERATORS IN UNIFORMLY CONVEX BANACH SPACES
}

\author{
HONG-KUN XU
}

(Communicated by Palle E. T. Jorgensen)

\begin{abstract}
Let $(\Omega, \Sigma)$ be a measurable space with $\Sigma$ a sigma-algebra of subsets of $\Omega$, and let $C$ be a nonempty, bounded, closed, convex, and separable subset of a uniformly convex Banach space $X$. It is shown that every multivalued nonexpansive random operator $T: \Omega \times C \rightarrow K(C)$ has a random fixed point, where $K(C)$ is the family of all nonempty compact subsets of $C$ endowed with the Hausdorff metric induced by the norm of $X$.
\end{abstract}

The purpose of this note is to prove the random version of the following celebrated deterministic result due to Lim [12].

Theorem L [12]. Let $X$ be a uniformly convex Banach space, let $C$ be a nonempty, bounded, closed, convex subset of $X$, and let $K(C)$ be the collection of all nonempty compact subsets of $C$ endowed with the Hausdorff metric. Then every multivalued nonexpansive mapping $T: C \rightarrow K(C)$ has a fixed point.

We begin with establishing some preliminaries. By $(\Omega, \Sigma)$ we denote a measurable space with $\Sigma$ a sigma-algebra of subsets of $\Omega$. Let $(X, d)$ be a metric space. Then we denote by $K(X)$ the family of all nonempty compact subsets of $X$ and by $H$ the Hausdorff metric on $K(X)$ induced by the metric $d$ of $X$; that is, for $A, B$ in $K(X)$,

$$
H(A, B)=\max \left\{\sup _{a \in A} d(a, B), \sup _{b \in B} d(b, A)\right\},
$$

where $d(x, E)$ is the distance from a point $x \in X$ to a subset $E \subset X$, i.e., $d(x, E)=\inf \{d(x, y): y \in E\}$. Recall that a multivalued mapping $T: X \rightarrow$ $K(X)$ is said to be nonexpansive if

$$
H(T x, T y) \leq d(x, y)
$$

for all $x, y$ in $X$ and that a point $x$ in $X$ is said to be a fixed point of $T$ if $x \in T x$. A multivalued operator $f: \Omega \rightarrow X$ is said to be $(\Sigma$-)measurable if, for any open subset $B$ of $X, f^{-1}(B)=\{\omega \in \Omega: f(\omega) \cap B \neq \varnothing\} \in \Sigma$. Let $M$ be

Received by the editors August 1, 1991.

1991 Mathematics Subject Classification. Primary 47H10, 47H09; Secondary 60H25.

Key words and phrases. Random fixed point, multivalued nonexpansive operator, uniformly convex Banach space. 
a nonempty closed subset of $X$. Then a multivalued operator $f: \Omega \times M \rightarrow X$ is called a random operator if, for each fixed $x \in M$, the map $f(\cdot, x): \Omega \rightarrow X$ is measurable. A single-valued operator $x: \Omega \rightarrow X$ is said to be a random fixed point of a multivalued random operator $f: \Omega \times M \rightarrow X$ if $x$ is measurable and $x(\omega) \in M \cap f(\omega, x(\omega))$ for all $\omega \in \Omega$. A multivalued random operator $f: \Omega \times M \rightarrow X$ is said to be continuous (resp. nonexpansive, etc.) if for each fixed $\omega \in \Omega$, the multivalued map $f(\omega, \cdot): M \rightarrow X$ is continuous (resp. nonexpansive, etc.). We shall use the notion of a "universal net."

A net $\left\{x_{\alpha}\right\}$ in a set $S$ is said to be universal if for each subset $U$ of $S$, either $\left\{x_{\alpha}\right\}$ is eventually in $U$ or $\left\{x_{\alpha}\right\}$ is eventually in $S \backslash U$. The following facts are pertinent $[8$, p. 81$]$ :

(a) Every net in a set has a universal subset.

(b) If $f: S_{1} \rightarrow S_{2}$ and $\left\{x_{\alpha}\right\}$ is a universal net in $S_{1}$, then $\left\{f\left(x_{\alpha}\right)\right\}$ is a universal net in $S_{2}$.

(c) If $S$ is compact and $\left\{x_{\alpha}\right\}$ is a universal net in $S$, then $\lim _{\alpha} x_{\alpha}$ exists. The reader is referred to Kirk [9], Kirk and Massa [10], Kirk and Yanez [11], and Aksoy and Khamsi [1] for applications of universal nets to fixed point theory.

We now state and prove the main result of this note, which can be viewed as the stochastic version of Lim's theorem mentioned above. See $[2,6,7$, $15,17]$ and references therein for more details of random fixed point theory. We note that previous existence results for random fixed points of multivalued nonexpansive operators are obtained only in a space that satisfies Opial's condition [16]. A Banach space $X$ is said to satisfy Opial's condition if for any sequence $\left\{x_{n}\right\}$ in $X$, the condition $x_{n} \rightarrow x \in X$ weakly implies that $\liminf _{n \rightarrow \infty}\left\|x_{n}-y\right\|>\liminf _{n \rightarrow \infty}\left\|x_{n}-x\right\|$ for all $y \in X, y \neq x$. It is known [16] that all Hilbert spaces and the $l^{p}$ spaces $(1<p<\infty)$ enjoy this property; however, the $L^{p}$ spaces do not unless $p=2$.

Theorem. Let $(\Omega, \Sigma)$ be a measurable space with $\Sigma$ a sigma-algebra of subsets of $\Omega$, let $C$ be a nonempty, bounded, closed, convex, and separable subset of a uniformly convex Banach space $X$, and let $T: \Omega \times C \rightarrow K(C)$ be a multivalued nonexpansive random operator. Then $T$ has a random fixed point.

Proof. Let $v \in C$ be fixed and set, for each integer $n \geq 1$,

$$
T_{n}(\omega, x)=\left(1-\frac{1}{n}\right) T(\omega, x)+\frac{1}{n} v, \quad \omega \in \Omega, x \in C .
$$

Then each $T_{n}$ is a multivalued random contraction and hence has a random fixed point $x_{n}(\omega)$ by Itoh [6]. Let $\left\{n_{\alpha}\right\}$ be a universal subnet of the positive integers $\{n\}$. Define a function $f: \Omega \times C \rightarrow R^{+}:=[0, \infty)$ by

$$
f(\omega, x)=\lim _{\alpha}\left\|x_{n_{u}}(\omega)-x\right\| \text {. }
$$

Since $\left\{x_{n_{\alpha}}(\omega)\right\}$ is countable, it is easily seen that $f(\omega, x)$ is measurable. On the other hand, since the space $X$ is uniformly convex and $C$ is weakly compact convex, there exists a unique point $x(\omega) \in C$ such that

$$
f(\omega, x(\omega))=\inf _{x \in C} f(\omega, x)=: r(\omega) .
$$

This point $x(\omega)$ is referred to as, according to terminology of Edelstein [4] and Lim [14], the asymptotic center of the net $\left\{x_{n_{t}}(\omega)\right\}$ in $C$. Lim [13], Goebel 
[5], and Kirk and Massa [10] actually proved that for each fixed $\omega \in \Omega, x(\omega)$ is a fixed point of the map $T(\omega, \cdot)$. So to complete the proof of the theorem, it remains only to show the measurability of $x(\cdot)$. To this end, let $\left\{u_{n}\right\}_{n=1}^{\infty}$ be a countable dense subset of $C$. (This can be done since $C$ is separable.) Then one easily sees that $r(\omega)=\inf _{n \geq 1} f\left(\omega, u_{n}\right)$. We first show that $r: \Omega \rightarrow R^{+}$is measurable. In fact, for every real number $s$, we have

$$
\begin{aligned}
\{\omega \in \Omega: r(\omega) \geq s\} & =\left\{\omega \in \Omega: \inf _{n \geq 1} f\left(\omega, u_{n}\right) \geq s\right\} \\
& =\bigcap_{n=1}^{\infty}\left\{\omega \in \Omega: f\left(\omega, u_{n}\right) \geq s\right\} \in \Sigma
\end{aligned}
$$

since $f$ is measurable. This proves the measurability of $r(\omega)$. Now for each integer $k \geq 1$, we let

$$
A_{k}(\omega)=\left\{x \in C: f(\omega, x) \leq r(\omega)+\frac{1}{k}\right\} .
$$

It then follows that $A_{k}: \Omega \rightarrow C$ is measurable and for each $\omega \in \Omega, A_{k}(\omega)$ is a weakly compact convex subset of $C$. It is readily seen that

$$
\bigcap_{k=1}^{\infty} A_{k}(\omega)=\{x(\omega)\}
$$

Since $C$ is separable, the weak topology on $C$ is metrizable. Let $d_{w}$ be the metric produced by the weak topology on $C$ and let $H_{w}$ be the Hausdorff metric induced by $d_{w}$. We show that

$$
\lim _{k \rightarrow \infty} H_{w}\left(A_{k}(\omega), x(\omega)\right)=0 .
$$

Indeed, since $\bigcap_{k=1}^{\infty} A_{k}(\omega)=x(\omega)$ by (1), the limit in (2) exists and we denote it by $h(\omega)$. If $h(\omega)>0$, then observing

$$
H_{w}\left(A_{k}(\omega), x(\omega)\right)=\sup \left\{d_{w}(y, x(\omega)): y \in A_{k}(\omega)\right\},
$$

we have for each $k$ a $y_{k} \in A_{k}(\omega)$ such that

$$
d_{w}\left(y_{k}, x(\omega)\right)>\frac{1}{2} h(\omega) .
$$

Since $C$ is weakly compact, there is a subsequence $\left\{y_{k^{\prime}}\right\}$ of $\left\{y_{k}\right\}$ converging weakly to some $y \in C$, i.e., $d_{w}\left(y_{k^{\prime}}, y\right) \rightarrow 0$ as $k^{\prime} \rightarrow \infty$. Since $\left\{A_{k}(\omega)\right\}$ is decreasing, $y \in \bigcap_{k=1}^{\infty} A_{k}(\omega)=x(\omega)$. It thus follows that $d_{w}\left(y_{k^{\prime}}, x(\omega)\right) \rightarrow 0$ as $k^{\prime} \rightarrow \infty$. This contradicts (3), and (2) is proven. By Itoh [6, Proposition 1], it follows that $x(\omega)$ is weakly measurable, i.e., for every $x^{*} \in X^{*}$, the dual space of $X, x^{*} x(\omega)$ is measurable as a numerically valued function on $\Omega$. Again since $C$ is separable, by Bharucha-Reid [3, Theorem 1.2], $x(\omega)$ is measurable. The proof of the theorem, therefore, is complete.

\section{ACKNOWLEDGMENT}

The author is grateful to Professor Jia-Gang Wang for his valuable discussions. 


\section{REFERENCES}

1. A. G. Aksoy and M. A. Khamsi, Nonstandard methods in fixed point theory, Springer-Verlag, New York, Berlin, and Heidelberg, 1990.

2. A. T. Bharucha-Reid, Fixed point theorems in probabilistic analysis, Bull. Amer. Math. Soc. 82 (1976), 641-645.

3. _ Random integral equations, Academic Press, New York and London, 1972.

4. M. Edelstein, The construction of an asymptotic center with a fixed point property, Bull. Amer. Math. Soc. 73 (1972), 206-208.

5. K. Goebel, On a fixed point theorem for multivalued nonexpansive mappings, Ann. Univ. Mariae Curie Skłodowska Sect. A 29 (1975), 69-71.

6. S. Itoh, A random fixed point theorem for a multivalued contraction mapping, Pacific J. Math. 68 (1977), 85-90.

7. __ Random fixed point theorems with an application to random differential equations in Banach spaces, J. Math. Anal. Appl. 67 (1979), 261-273.

8. J. L. Kelly, General topology, van Nostrand, Princeton, NJ, 1955.

9. W. A. Kirk, An iteration process for nonexpansive mappings with applications to fixed point theory in product spaces, Proc. Amer. Math. Soc. 107 (1989), 411-415.

10. W. A. Kirk and S. Massa, Remarks on asymptotic and Chebyshev centers, Houston J. Math. 16 (1990), 357-364.

11. W. A. Kirk and C. Martinez Yanez, Nonexpansive and locally nonexpansive mappings in product spaces, Nonlinear Anal. 12 (1988), 719-725.

12. T. C. Lim, A fixed point theoren for multivalued nonexpansive mappings in a uniformly convex Banach space, Bull. Amer. Math. Soc. 80 (1974), 1123-1126.

13. _ Remarks on some fixed point theorems, Proc. Amer. Math. Soc. 60 (1976), 179-182.

14. __ Characterization of normal structure, Proc. Amer. Math. Soc. 43 (1974), 313-319.

15. T. C. Lin, Random approximations and random fixed point theorems for non-self-maps, Proc. Amer. Math. Soc. 97 (1986), 507-514.

16. Z. Opial, Weak convergence of the sequence of successive approximations for nonexpansive mappings, Bull. Amer. Math. Soc. 73 (1967), 595-597.

17. H. K. Xu, Some random fuxed point theorems for condensing and nonexpansive operators, Proc. Amer. Math. Soc. 110 (1990), 395-400.

Institute of Applied Mathematics, East China University of Chemical Technology, Shanghai 200237, China 